\title{
When Memory Shifts Toward More Typical Category Exemplars: Accentuation Effects in the Recollection of Ethnically Ambiguous Faces
}

\author{
Olivier Corneille ${ }^{1}$, Johanne Huart ${ }^{2}$, Emilie Becquart $^{2}$, Serge Brédart ${ }^{3}$ \\ ${ }^{I}$ Department of Psychology, Catholic University of Louvain, Louvain-la-Neuve, Belgium, and the Fonds National de la Recherche \\ Scientifique, Brussels, Belgium; \\ ${ }^{2}$ Department of Psychology, Catholic University of Louvain; \\ ${ }^{3}$ Department of Psychology, University of Liège, Liège, Belgium.
}

In 4 studies, the authors examined the impact of categorization on the recollection of ethnically ambiguous faces. Participants were presented with faces lying at various locations on mixed-race continua (i.e., Caucasian-North African and Caucasian-Asian faces were used as source images in a morphing program). In all studies, the prevalence of exclusive ethnic features in a face distorted participants' recollections of the face toward faces more typical of the category. Specifically, the recollection of 30\% North African (or 30\% Asian) faces shifted toward Caucasian source faces, whereas the recollection of $70 \%$ North African (or $70 \%$ Asian) faces shifted toward North African (Asian) source faces. Memory distortions did not emerge for extremely ambiguous $(50 \%)$ faces and proved larger on mixed-race than same-race continua (Studies 3 and 4). Memory distortions also emerged with high levels of confidence. The authors elaborate on the theoretical and practical implications of these findings.

Have you ever noticed, after meeting a person you have not seen for a period of time, that this person looked different than you remembered? Last year, an Italian colleague of ours returned from a 2 -week holiday back in his homeland. As he entered the lab and greeted us, we commented on our feeling that his hair and eyes were actually lighter than we remembered. The sun may have lightened his hair during his holidays, but what explanation could hold for his eyes? Was our recollection of his face simply inaccurate? We speculated that within only 2 weeks, our memory of our colleague's face had shifted toward a face holding more typical characteristics of his national group.

This event nicely illustrates the effect investigated here. Indeed, we hypothesized that face memory would be distorted toward more typical face exemplars. Although this issue could be addressed in the context of various categories (e.g., age, gender), we focused on ethnic categories in the present research. We hypothesized that the spontaneous categorization of ethnically ambiguous faces would result in memory distortions of these faces toward more typical face exemplars. The influence of social information externally attached to the faces was also considered.

\section{Practical Implications of Category-Based Distortions of Face Memory}

Most (if not all) people encountered in everyday life display mixed ethnic features. People differ, however, regarding the extent to which they elicit the notion of ethnic typicality. Although the ethnic typicality of a fece has been shown to influence people's affective and semantic reactions to that face (e.g., Blair, Judd, Sadler, \& Jenkins, 2002; Livingston \& Brewer, 2002; Maddox \& Gray, 2002), very little is known about how ethnically ambiguous faces are recollected from memory (for a recent exception, see Eberhardt, Dasgupta, \& Banaszynski, 2003). A better understanding of this issue may prove relevant to eyewitness testimony.

One of the most extensively studied biases in the eyewitness testimony literature is the other-race effect. This bias refers to people's lower accuracy in recognizing other-race versus own-race faces (Malpass \& Kravitz, 1969). This effect is critical in courtroom trials and criminal investigations that involve suspects and eyewitnesses whose races differ. Most other-race effect studies have involved faces whose ethnic identity was obvious. Virtually nothing is known, however, about biases arising in the recollection of ethnically ambiguous faces. To the best of our knowledge, only MacLin and Malpass (2001) examined memory biases in the context of such faces. These authors found that people process subtle ethnic markers (e.g., hairstyle) to decode the ethnicity of ambiguous faces. This categorization process impacts on the perception of the faces (e.g., the same mouth will be perceived as wider if the face is associated with a Black rather than a Hispanic hairstyle) and may result in category-consistent confusions at the recollection stage (e.g., the face, if categorized as Black, will more likely be confused later on with Black than with Hispanic foils). Studies by MacLin and Malpass (2001) have extended understanding of categorization effects in face memory. These studies, however, did not address 
whether memory is distorted toward more race-typical faces. Of importance, too, this work did not examine the roles that either ethnic labels or stereotypical information externally attached to a face may play in the recollection process. Evidence obtained on the latter issue would extend knowledge of identification biases in significant ways. For instance, do people recall ethnically ambiguous faces (e.g., light-skinned African Americans) as more typical of their category (e.g.. darker skinned) than they actually are? Does stereotypical information associated with ethnically ambiguous faces (e.g., the trait "violent" associated with a light-skinned African American) result in memory distortions consistent with this information (e.g., distortion toward a darker skinned face)? If these distortions emerge, are they equivalent for own-race and other-race identifications? Or, alternatively, given (a) the other-race effect, (b) the higher complexity with which own-race faces are attended to (Levin, 2000), and (c) the higher adequacy of the dimensions on which own-race faces are represented in memory (Valentine, 1991), are memory distortions toward more typical faces smaller when recollecting ownrace than other-race faces? The present research directly addressed these issues.

\section{Theoretical Perspectives on Category-Based Distortions of Face Memory}

Social psychologists have paid little attention to the influence of categorization on memory confusions about group members. A noteworthy exception to this is the work initiated by Taylor, Fiske, Etcoff, and Ruderman (1978) using the "who-said-what" paradigm. Consistent with the classic accentuation effect evidenced by Tajfel and Wilkes (1963; see also Corneille, Klein, Lambert, \& Judd, 2002), this research demonstrated that people are more likely to misremember face-statement associations within than between group members (e.g., Klauer \& Wegener, 1998; Taylor et al., 1978). The who-said-what research program revealed the impact of categorization on people's memory for face-statement associations. Taylor et al.'s work, however, did not address the impact of categorization on the recollection of face features.

Very little cognitive or social psychological work has actually examined the impact of categorization on memory distortions toward more typical faces. In the context of face memory, Halberstadt and Niedenthal (2001) have provided recent evidence that faces conceptualized in terms of anger or happiness are recollected as angrier or happier than they are. In these studies, the effects resulted from an explicit categorization of the emotion displayed by the face. Consistent with earlier work showing memory interferences due to verbalization (e.g., Dodson, Johnson, \& Schooler, 1997), memory distortions emerged as participants provided an explanation for the categorical information attached to a face while they were attending to the face.

In the present research, we examined whether memory distortions would emerge during recall of ethnically ambiguous faces. More specifically, we tested for the accentuation of face memory toward faces more typical of the category. We examined undirected and directed categorization effects in producing memory distortions. These refer, respectively, to effects resulting from features inherent in a face (undirected categorization) and from social information externally attached to a face (directed categorization).

For the undirected categorization effects, we anticipated that participants would spontaneously categorize moderately ambiguous faces according to their inherent and predominant ethnic features. We expected that this process would lead to memory distortions toward more typical faces. This prediction was based on the general notion that people rely on category and schematic information when reconstructing inexact memories. In particular, Huttenlocher and colleagues (Huttenlocher, Hedges, \& Duncan, 1988; Huttenlocher, Hedges, \& Vevea, 2000; but see also Bartlett, 1932; Brewer \& Nakamura, 1984; Fiedler, 1993; Fiedler, Armbruster, Nickel, Walther, \& Asbeck, 1996) posited that people encode fine-grained (i.e., perceptual) information along with selfgenerated categorical information about visual stimuli. Because fine-grained representations are generally poor, people combine these with self-generated categorical information when asked for stimuli recollections, a process Huttenlocher et al. $(1988,2000)$ called weighting with a prototype. The recollections are adjusted toward the most typical (prototypical) values of the category, which are spontaneously computed as participants process discrete category exemplars (Posner \& Keele, 1968; in the context of face memory models, see also Valentine, 1991). This model has been successfully tested on relatively simple materials (for example, the localization of dots in a circle), but it is defined as a general model of categorization effects on reports from episodic memory. It may therefore be generalized to more complex materials.

As for the directed categorization effects, much more akin to Halberstadt and Niedenthal's (2001) work, predictions were more uncertain. We anticipated that participants would not rely on categorical information externally attached to a face when examining a moderately ambiguous face. As just explained, we hypothesized that participants would spontaneously categorize these faces on the basis of their predominant features. Social information highly consistent with this spontaneous categorization process should be deemed redundant and should therefore add little to categorization effects. Alternatively, external information inconsistent with the 
spontaneous categorization of these faces should be deemed unlikely and may, therefore, be disregarded.

For extremely ambiguous faces, two patterns of results could emerge. Consistent with the person perception literature, people may actively seek out external information in order to make sense of the identity of a very ambiguous face (for a related point, see Trope, 1986; Trope \& Cohen, 1989; Trope, Cohen, \& Maoz, 1988). This process may lead to categorizing an extremely ambiguous face in line with the social information attached to it. This may, in turn, result in category-consistent distortions. For instance, a perfect mix between an Asian and a Caucasian face may be categorized as Asian or Caucasian when the social information suggests that this person is Asian or Caucasian, resulting in memory distortions toward more typical Asian or Caucasian faces.

Alternatively, participants may have belongs to either category implied by extremely ambiguous face belongs to either category implied by the social information attached to it In our example, people may be reluctant to accept that a perfect mix between an Asian and a Caucasian person belongs to either of these categories. If so, this information should be deemed inapplicable to the face, and it should therefore play little role in the recollection process (for a general discussion on applicability effects in person perception, see Higgins, 1996). ${ }^{1}$

In sum, two issues were addressed in the present research. First, we examined whether undirected categorization effects would result in memory distortions toward more typical faces in recollecting moderately ambiguous ethnic faces. On the basis of previous work on reconstructive memory, we predicted that this would indeed be the case. Second, we examined whether the recollection of ethnically ambiguous faces would be sensitive to a directed categorization process. We anticipated that such an effect would not emerge for moderately ambiguous faces. As for extremely ambiguous faces, predictions were more uncertain.

\section{Overview of the Studies}

We examined the impact of the directed (Studies 1 and 2) and undirected (Studies 1-4) categorization of faces on the recollection of these faces. In all studies, participants were exposed to faces that varied with regard to ethnic typicality, some of which were associated with social information (Study 1) or ethnic labels (Study 2). Following their exposure to the faces, participants took part in a distraction task immediately followed by an unexpected identification task. The later task was designed so that it allowed for evidence recollection distortions toward faces more typical of the category.

Studies 1 and 2 were primarily focused on the impact of undirected and directed categorization processes on the recollection of ethnically ambiguous faces. Studies 3 and 4 provide evidence that the memory distortions obtained in Studies 1 and 2 consisted of memory shifts toward more typical faces instead of shifts toward distinctive faces.

\section{Study 1}

\section{Method Participants and Design}

Ninety-six Caucasian undergraduate students at the Catholic University of Louvain took part in the experimental sessions in return for partial course credit. They participated in groups of 1 to 4 people and were randomly assigned to one of six conditions defined by a Social Information (typical of Caucasian vs. North African people) $\times$ Morphing (faces were $30 \%$ vs. $50 \%$ vs. $70 \%$ North African) between-participants design.

\section{Materials}

Four Caucasian-North African continua were created, using a morphing program (Morph TM 2.5; Gryphon Software Corporation, 1992-1994). The source faces were selected from a great number of people photographed on the street, who gave their agreement for using their pictures in this research. Eight faces were selected on the basis of good results in pretests regarding their ethnic category, so that each Caucasian or North African source face was clearly perceived as being Caucasian or North African. The pictures were cleaned so that their backgrounds were totally white and their sizes were equalized.

From this material, we generated target faces that were located at either $30 \%, 50 \%$, or $70 \%$ of morphing on the four different Caucasian-North African continua. Four distractors were built up for the first identification task,

\footnotetext{
${ }^{1}$ A recent study by Eberhardt et al. (2003) suggested that the impact of social information externally attached to ambiguous ethnic faces may actually depend on the perceivers' naive theories. We come back to this point in the General Discussion section.
} 
which were located $10 \%$ and $20 \%$ closer than the target faces to the Caucasian and North African source faces. So in the $50 \%$ condition, the distractors were located at $30 \%, 40 \%, 60 \%$, and $70 \%$ on the Caucasian-North African continua. In the $30 \%(70 \%)$ conditions, they were located at $10 \%, 20 \%, 40 \%$, and $50 \%(50 \%, 60 \%$, $80 \%$, and $90 \%$ ) on the Caucasian-North African continua. The distractors and the target faces were printed out in a gray mode on a white sheet of paper. They were used in a card format, which could easily be randomly presented during the recognition task. An example of a Caucasian-North African continuum can be seen in Appendix A.

The social information associated with the faces consisted of a name typical of either a North African person (i.e., Mohammed) or a Caucasian person (i.e., Benoît), along with eight statements pretested for valence and typicality. Statements were neutral in valence. Four statements were ethnically irrelevant and acted as fillers (e.g., "eats sandwiches for lunch"). The remaining statements were pretested so as to be highly and exclusively typical of either a person of Belgian origin (e.g., "makes few gestures when speaking") or North African origin (e.g., "sometimes goes to the Mosque").

\section{Procedure}

On their arrival at the laboratory, participants were greeted by the experimenter and were given an experimental booklet. Participants were asked to proceed one page at a time without going back and forth throughout the booklet. The procedure consisted of a learning task, a distraction task, and an unexpected identification task followed by a categorization task. Once the experimental session was completed, participants were thanked and invited to sign up for a follow-up study, which was to take place 1 week later.

Learning task. The study was presented as an investigation on impression formation. Participants were presented with one target face, which they were instructed to examine attentively. Social information about the face was delivered on the next page ${ }^{2}$ in a random order, including the name of me target (typical of either a North African or Caucasian person) along with eight statements (four of them neutral and four of them typical either of a North African or Caucasian person). Participants were asked to read this information carefully because they would need to use it later on to complete the impression formation task.

Distraction task. Participants read an article about city traffic and completed a questionnaire on this article. Then, they completed an impression formation task in which they were asked to describe a typical day in the target's life. This was done to ensure that participants would process the social information they had received about the target. After that point, neither the picture nor the social information delivered about the target was communicated to the participants again.

Unexpected identification task Participants were unexpectedly presented with five faces: the target face and the four distractors. The faces were presented simultaneously to the participants and were arranged in a random pattern. Participants were asked to single out the face they had previously seen among the five pictures and to report how confident they were in their choice (from $0 \%=$ Not at all confident to $100 \%=$ Totally confident).

Categorization of the face. Once the recognition task was completed, participants were asked to report how they had spontaneously categorized the face. This information was collected in order to examine whether associations could be found between the spontaneous categorization of the face at Time 1 and the recollection of the face at Time 1 and 1 week later (see below). ${ }^{3}$

Identification at Time 2. Participants were invited to return to the laboratory 1 week later to take part in a followup study. Nearly $75 \%$ of the participants (71 out of 96) returned for the second session. They were presented with two distractors, one face that was $10 \%$ closer than the target face to the Caucasian source face and one face that was $10 \%$ closer to the Norm African source face. We did not include the other three pictures in the identification set (the target face and the two extreme Caucasian and North African distractors) in order to gain power in examining the associations between categorization at Time 1 and distortions at Time 2.

\footnotetext{
${ }^{2}$ In Study 1, social information was delivered after participants were exposed to the faces. In Study 2, social information consisted of an ethnic label mat was located below the faces. In an unreported replication of Study 1, conducted on 38 participants, social information was delivered before participants were exposed to $50 \%$ of the faces. Similar findings were obtained in all three studies regarding the impact of social information on the recollection process.

${ }^{3}$ We chose not to take categorization measures before the identification task in order to avoid possible contaminations on the recollection process. Indeed, we feared that within the same experimental session participants would consciously match their identification decision with their prior explicit categorization of the face (see the "postevent questioning effect"; Loftus, 1975; Loftus, Miller, \& Bums, 1978; Loftus \& Palmer, 1974; Shaw, 1996).
} 
Published in: Journal of Personality and Social Psychology (2004), vol. 86, iss. 2, pp. 236-250.

Status: Postprint (Author's version)

Participants were fully debriefed in a collective information session when the data had been collected.

Table 1 Mean Recollection Distortion of Caucasian-North African Faces as a Function of Level of Morphing (Study 1)

\begin{tabular}{lccc}
\hline & \multicolumn{3}{c}{ Level of morphing (\%) } \\
\cline { 2 - 4 } Recollection distortion & 30 & 50 & 70 \\
\hline$M$ & -0.60 & 0.00 & 0.55 \\
$S D$ & 1.13 & 1.00 & 0.85 \\
\hline
\end{tabular}

Note. Values range from -2 (20\% distortion toward the Caucasian source face) to +2 (20\% distortion toward the North African source face)

\section{Results}

\section{Identification and Confidence at Time 1}

The error rate was high: Only 35 out of 96 participants selected the correct face. Participants' answers were recoded in terms of distortions from the target face, with values of 0 for correct identifications; values of -1 and 2 , respectively, for distortions $10 \%$ and $20 \%$ toward the Caucasian or North African source face; and values of +1 and +2 , respectively, for distortions $10 \%$ and $20 \%$ toward the North African source face. As predicted, a 2 (social information) $\times 3$ (morphing) between-participants analysis of variance $($ ANOVA) revealed a main effect of the morphing factor on participants' recollections, $F(2,95)=10.54, p=.01$. Specifically, identifications were distorted toward the Caucasian source face in the $30 \%$ North African condition $(M=-0.60, S D=1.13), F(1,31)$ $=9.81, p<.01$, and toward the North African source face in the 70\% North African condition $(M=0.55, S D=$ $0.85), F(1,30)=12.9, p<.01$. In the $50 \%$ condition, no systematic distortion emerged $(M=0.00, S D=1.00$; see Table 1). No main or moderating impact of the social information factor was evidenced on participants' recollections $(F s<1)$

We also examined associations between participants' categorization reports and memory distortions at Time 1 . We considered associations as assimilative when the recollection of a face categorized as Belgian or North African was distorted toward the Caucasian or North African source face. We considered associations as contrastive in the opposite cases. As predicted, assimilative associations $(n=32)$ outnumbered contrastive associations ( $n=20$; binomial, $p=.06$; we removed from the analyses participants whose recollections were correct or participants who did not report a Belgian or North African categorization at Time 1).

Finally, participants were quite confident in their identifications $(M=76.70, S D=16.21)$, irrespective of whether they made a correct or incorrect choice $(F<1)$. Confidence ratings were not influenced by the experimental factors or by their interaction $(F s<1)$.

\section{Identification at Time 2 as a Function of Categorization at Time 1}

It is important to note that at Time 2, participants were more likely to select a distractor that was consistent $(n=$ 38 ) than inconsistent $(n=23)$ with their spontaneous categorization of the face at Time 1 (binomial, $p<.04$; we removed from the analyses participants who did not report a Belgian or North African categorization at Time 1). Thus, participants' initial categorization of the face predicted memory distortions 1 week later.

\section{Discussion}

Results from this study fully support our predictions for moderately ambiguous (30\% and $70 \%)$ faces. Indeed, faces moderately typical of a Caucasian or North African person were misrecollected as more Caucasian or North African than they were. Under these conditions, the recollection process was insensitive to the social information attached to the faces. Also, no evidence was obtained for the influence of the social information on recollecting extremely ambiguous (i.e., 50\%) faces. This may suggest that information delivered about these faces did not match participants' face representations.

It is also important that participants' spontaneous categorization of the face at Time 1 predicted memory distortions both at Time 1 and Time 2, and fairly high levels of confidence were obtained in the present study both for correct and incorrect identifications. Given this finding, it is unlikely that participants simply guessed in 
the identification task. Rather, it seems that their memory traces, or recollections, of the faces were distorted toward more typical faces.

\section{Study 2}

Study 2 was aimed at examining whether findings obtained in Study 1 would generalize to other continua types and to situations where perceivers were presented with multiple faces at the encoding. In this second study, participants were sequentially presented with eight faces located either at a 30\%, 50\%, or $70 \%$ level of morphing on Caucasian-North African (four faces) and Caucasian-Asian (four faces) continua. Each of the faces was presented along with an ethnic label. Obtaining systematic recollection distortions under conditions in which several faces from multiple continua types are involved would substantially increase the generality of our findings.

Although the main goal of Study 2 was to test for generality in the recollection distortions obtained in Study 1 under the $30 \%$ and $70 \%$ conditions, it also sought to examine (through a different procedure) the possibility that social information influences the recollection of extremely ambiguous $(50 \%)$ faces. One possible reason why the latter effect did not emerge in Study 1 is that some participants corrected for mental contamination at the identification time. That is, some participants may have considered that the information initially attached to the face was biasing their identification decision. These participants may then have corrected for this influence by selecting a distractor that was inconsistent with the information initially associated with the face.

The visuolexical distraction task used in Study 2, because of the cognitive load it implied, precluded participants from explicitly recalling the face-label associations at the identification time. If the lack of impact of the social information obtained in Study 1 was due to corrective processes, then this effect might emerge in the present study. Clearly, people are not supposed to correct for a mental contamination they are not aware of (see, e.g., Wilson \& Brekke, 1994).

Our predictions for this second study were as follows. In the $30 \%$ and $70 \%$ conditions, we predicted that the effects obtained in Study 1 would be replicated for both continua types, with distortion toward the Caucasian source face in the $30 \%$ condition and distortions toward the North African or Asian source face in the $70 \%$ condition. We also explored the influence of social information externally attached to the extremely ambiguous $(50 \%)$ faces under the present conditions.

Although participants in Study 1 singled out one face among four distractors, this time they were presented with pairs of faces, each including the target face along with one distractor closer either to the Caucasian, North African, or Asian source face. The participants' task was to select the target face in the pair on each presentation. This procedure resulted in a total of 64 identification decisions concerning eight different continua instead of 1 decision on one continuum only. For practical reasons, and given the enhanced difficulty of the identification task, only one identification task was conducted in the present study. That is, the delayed memory test was dropped.

\section{Method}

\section{Participants and Design}

Forty-eight Caucasian undergraduate students at the Catholic University of Louvain took part in the experimental session on a voluntary basis. They participated in collective computer sessions of 6 to 14 people. The design was a 3 (morphing: $30 \%$ vs. $50 \%$ vs. $70 \%$ faces) $\times 4$ (association type: Caucasian-North African face with Caucasian label, Caucasian-North African face with North African label, Caucasian-Asian face with Caucasian label, and Caucasian-Asian face with Asian label) $\times 2$ (distractor type: target face along with a Caucasian vs. North African-Asian distractor) experimental design, with association type and distractor type as vwithin-participants factors. Participants were randomly assigned to one of the three levels of the morphing factor. That is, they were presented with either eight $30 \%$, eight $50 \%$ or eight $70 \%$ faces. Because we had four face exemplars for both the Caucasian-North African and Caucasian-Asian continua types, face-label associations were counterbalanced within continua types.

\section{Materials}

Eight continua were used. Four were the four Caucasian-North African continua used in Study 1, and four were new Caucasian-Asian continua created following a similar procedure. An example of a Caucasian-Asian 
continuum can be seen in Appendix B. The target faces were located at 30\%, 50\%, or $70 \%$ on the continua, and they were each presented with an ethnic label, which read "North African origin," "Asian origin," or "Belgian origin." Two Caucasian-North African and Caucasian-Asian faces were associated with the Caucasian label and two were associated with the North African or Asian label.

The distractors used in the identification pairs were $10 \%$ and $20 \%$ closer to the Caucasian and North AfricanAsian source images of the continua. This time, the faces were presented in color, except for two continua (one Caucasian-North African and one Caucasian-Asian) for which a color version was not available.

\section{Procedure}

On their arrival at the computer room, participants were seated in front of a Macintosh 7260/90 PowerPC computer with a Trinitron monitor. All instructions were communicated via the computer program. The experimental sessions included sequentially a learning task, a distraction task, and an unexpected identification task. Right after the identification task, participants were asked to recall the label initially associated with each lace and to report how they had spontaneously categorized the faces. These tasks are described below.

Learning task. The first part of the session was presented as an investigation of age estimates on the basis of ethnic information. Participants were sequentially presented with eight faces in a random order, each associated with an ethnic label. They were asked to pay close attention to the face and to the label information attached to it. After $8 \mathrm{~s}$, the face and the label disappeared from the screen and participants had to enter their estimate on the keyboard. Once their estimate was entered, a new stimulus appeared on the screen for $8 \mathrm{~s}$. We used that procedure to make sure that participants would pay attention to the label information and would relate this label to the face.

Distraction task. The distraction task consisted of learning associations between visual and verbal information. Pictures of 16 unfamiliar plants appeared thrice on the computer screen, each time for $4 \mathrm{~s}$, along with their name. These stimuli were presented in a random order, and participants were instructed to pay close attention to them because they would have to recall this information later on. A recognition task followed the presentation of the stimuli in which participants were presented with eight correct and eight incorrect picture-name associations. They had to indicate whether each association was correct or not. This task lasted for approximately $12 \mathrm{~min}$. Participants were led to believe that the task was aimed at evaluating their performance in processing visual information. We expected that this visuolexical task would erase participants' memory traces for the face-label associations, making this information unavailable to them at the recollection time.

Unexpected identification task. Participants were sequentially presented with pairs of faces in a random order, each including one target face from the eight continua along with one Caucasian or North African-Asian distractor from the same continuum. These pairs were presented twice, with the distractor appearing once at the right and once at the left of the target face. This procedure resulted in 64 identifications trials (eight continua $\times$ four distractors $x$ distractor presented at the left and at the right of the target face). On each presentation, participants were asked to indicate which of the two faces had been presented in the age estimate task and to report how confident they were in their judgment by pressing a key from 1 (Very unconfident) to 9 (Very confident). Note that the present procedure, in addition to bringing more power to the data analyses, allowed us (o examine independently memory distortions emerging toward both source faces.

Recall of the ethnic label information and reports on the categorization of the face. Once the identification task was completed, participants were presented with the target faces and were asked (a) to report the ethnic label previously attached to these faces and (b) to indicate how they had spontaneously categorized these faces.

Participants were thanked and fully debriefed in a collective information session once the data had been collected. 
Table 2 Mean Recollection Distortion of Mixed-Race Faces as a Function of Level of Morphing and Distractor Type (Study 2)

\begin{tabular}{lccc} 
& \multicolumn{3}{c}{ Level of morphing (\%) } \\
\cline { 2 - 3 } Distractor type & 30 & 50 & 70 \\
\hline Caucasian & 0.78 & 0.50 & 0.37 \\
$M$ & 0.16 & 0.19 & 0.19 \\
$S D$ & & & \\
North African and Asian & 0.21 & 0.49 & 0.67 \\
$M$ & 0.15 & 0.15 & 0.26 \\
SD & & & \\
\hline Note. Values range from 0 (correct identifications on all identification trials) to 1.5 (incorrect identifications on all identification trials).
\end{tabular}

\section{Results Identifications}

The percentage of errors (36\%) was somewhat lower than that obtained in Study 1. This difference is possibly due to a familiarity effect emerging throughout the identification task. Because the target faces were presented eight times and each distractor only twice in the identification task, the target faces became quite familiar to participants throughout the task. This familiarity may have increased the perceptual fluency of these exemplars and attracted participants' identification decisions (e.g., Begg, Anas, \& Farinacci, 1992; Reber \& Schwarz, 1999). In addition, because half of the pairs involved comparisons with $20 \%$ distractors (which lend themselves to fewer errors than $10 \%$ distractors do), the mean number of errors was smaller here than in Study 1 , which relied on a single identification.

Participants' answers were recoded to give evidence of memory distortions on both distractor types. For each of the four Caucasian-North African and the four Caucasian-Asian continua, participants answered to eight identification trials, four of them involving distractors that were $10 \%$ and $20 \%$ closer to the Caucasian source face and four of them involving distractors that were $10 \%$ and $20 \%$ closer to the North African-Asian source face. Selections of the target face in one identification trial were given a value of 0 on the distortion index, whereas selections of the $10 \%$ and $20 \%$ distractors were respectively recoded into values of 1 and 2 on this index. For each face, this procedure resulted in scores ranging from 0 (four correct identifications) to 6 (two selections of a $10 \%$ distractor and two selections of a $20 \%$ distractor). These scores, obtained separately for pairs involving both distractor types, were then averaged across faces, resulting in two distortion scores (toward the $0 \%$ and toward the $100 \%$ source faces) ranging from 0 (always correct) to 1.5 (always wrong).

The ethnic labels attached to the faces did not play any main or moderating effect on the distortion index $(F s<$ 1). In particular, ethnic labels did not influence participants' recollection of $50 \%$ faces. As expected, participants had a very difficult time recalling the face-label associations. The mean number of correct recollections (out of a maximum of eight) was $2.06(S D=2.41)$. Of the 48 participants, 25 failed to correctly recall any association. The lack of impact of the label, along with the poor recall of the label information at the identification time, suggests that the absence of effect of the context information should not be attributed to corrective processes.

Because the ethnic label was not an influence, for the sake of clarity this factor was dropped from the analyses Participants' identifications were submitted to a new 3 (morphing: $30 \%$ vs. $50 \%$ vs. $70 \%$ ) $\times 2$ (distractor type: Caucasian vs. North African-Asian) $\times 2$ (continua type: Caucasian-North African vs. Caucasian-Asian) mixed ANOVA with repeated measures on the last two factors. This analysis showed that distortions were stronger on Caucasian $(M=0.54, S D=0.24)$ than North African-Asian $(M=0.47, S D=0.26)$ distractors, $F(1,45)=4.18, p$ $<.05$. This effect was moderated by the continua type: Distortions on pairs involving a Caucasian distractor were stronger for Caucasian-Asian faces $(M=0.55, S D=0.32)$ than Caucasian-North African faces $(M=0.53, S D=$ $0.25), F(1,45)=5.89, p<.02$.

More importantly, we obtained support for our prediction that memory distortions would depend on the level of morphing of the faces. The Morphing $\times$ Distractor Type interaction was $F(2,45)=29.5, p<.01$. Specifically, for $30 \%$ faces, distortions were stronger on Caucasian than North African-Asian distractors, $F(1,45)=62.42, p<$ .01 . Conversely, for $70 \%$ faces, distortions were stronger on North African-Asian than Caucasian distractors, $F(1,45)=9.74, p<.01$. As for $50 \%$ faces, distortions were equivalent on both distractor types $(F<1$; see Table 2). 
A Morphing $\times$ Distractor Type $\times$ Continua Type interaction also emerged, $F(2,45)=8.54, p<.01$, with the Morphing $\times$ Distractor Type interaction found to be stronger for the Caucasian-Asian feces, $F(2,45)=38.61, p<$ .01 , than for the Caucasian-North African faces, $F(2,45)=8.59, p<.01$. The distortion profiles were the same on both continua types, but they were more pronounced on the Caucasian-Asian than die Caucasian-North African continua. Also, participants selected marginally more North African than Caucasian distractors when presented with 70\% Caucasian-North African faces, whereas they selected significantly more Asian than Caucasian faces when presented with 70\% Caucasian-Asian faces (see Table 3).

Finally, and also important, further analyses revealed that memory distortions toward more typical faces were stronger for the $30 \%$ faces than for the $70 \%$ faces, $F(1,28)=4.79, p<.04$. This effect may suggest greater distortions in recollecting own-race than other-race faces (the $0 \%$ source face always corresponded to a Caucasian face, and the participants were all Caucasians), and it did not appear to be moderated by the continua type (North African or Asian), $F(1,28)=1.35, p=.25$.

We also analyzed associations between participants' reported spontaneous categorization of the faces and their recollections of these faces. For each face, we computed an association index based on distortion scores for category-consistent and category-inconsistent memory distortions on that face. When category-consistent distortion scores were larger (smaller) than category-inconsistent distortion scores, a value of 1 ( -1 ) was given to the association index. For each participant, association scores were then averaged across all faces, with positive values reflecting a tendency toward category-consistent errors. As was the case for Study 1, associations between categorizations and distortions were significantly more assimilative than contrastive $(M=0.23, S D=0.45), F(1$, 46) $=12.03, p=.0011$.

Table 3 Mean Recollection Distortion of Mixed-Race Faces as a Function of Level of Morphing, Distractor Type, and Continua Type (Study 2)

\begin{tabular}{|c|c|c|c|}
\hline \multirow[b]{2}{*}{ Distractor type } & \multicolumn{3}{|c|}{ Level of morphing (\%) } \\
\hline & 30 & 50 & 70 \\
\hline & \multicolumn{3}{|c|}{ Continua type: Caucasian-North African } \\
\hline \multicolumn{4}{|l|}{ Caucasian } \\
\hline$M$ & 0.66 & 0.47 & 0.48 \\
\hline$S D$ & 0.22 & 0.23 & 0.26 \\
\hline \multicolumn{4}{|l|}{ North African } \\
\hline$M$ & 0.30 & 0.58 & 0.68 \\
\hline \multirow[t]{2}{*}{$S D$} & 0.16 & 0.19 & 0.33 \\
\hline & \multicolumn{3}{|c|}{ Continua type: Caucasian-Asian } \\
\hline \multicolumn{4}{|l|}{ Caucasian } \\
\hline$M$ & 0.90 & 0.53 & 0.27 \\
\hline$S D$ & 0.18 & 0.22 & 0.20 \\
\hline \multicolumn{4}{|l|}{ Asian } \\
\hline$M$ & 0.12 & 0.40 & 0.67 \\
\hline$S D$ & 0.14 & 0.18 & 0.37 \\
\hline
\end{tabular}

Note. Values range from 0 (correct identifications on all identification trials) to 1.5 (incorrect identifications on all identification trials).

\section{Classification of the Faces}

For each participant, we computed the percentage of time the faces were categorized toward the high end (i.e., Asian or North African) versus low end (i.e., Caucasian) of the continuum. As expected, this percentage was highly sensitive to the level of morphing, $F(2,44)=16.03, p<.01$, with more categorizations toward the high end category for the $70 \%$ faces $(M=0.79, S D=0.17)$ than for the $50 \%$ faces $(M=0.59, S D=0.19)$ and the $30 \%$ faces $(M=0.37, S D=0.24)$. Consistent with the findings obtained for the identification data, this effect was qualified by a Morphing $\times$ Continuum Type interaction, $F(2,44)=7.67, p<.01$, with a stronger impact of the morphing factor on the classification of Caucasian-Asian, $F(2,44)=28.89, p<.01$, than Caucasian-North African, $F(2,44)=2.36, p=.11$, faces. Importantly, however, additional analyses confirmed the existence of the expected linear trend on both the Caucasian-Asian, $F(1,44)=56.4, p<.01$, and Caucasian-North African, $F(1$, $44) \approx 4.69, p<.04$, continua of faces (see Table 4 for the full pattern of results). 


\section{Confidence}

Participants appeared to be relatively less confident in their identifications in Study 2 than in Study $1(M=4.82$, $S D=1.62$ ). Given the difficulty of the present task (eight different faces had to be identified several times among four different distractors, after a 12-min visual distraction task that prevented participants from rehearsing the original information), these levels of confidence are far from negligible.

Interestingly, participants in the present study appeared to be more confident when providing correct identifications $(M=5.00, S D=1.60)$ than incorrect identifications $(M=4.49, S D \approx 1.67), F(1,45)=37, p<.01$. We attribute this effect to the greater familiarity with the target face that emerged throughout the identification task (each target face was presented eight times relative to two times for any distractor). This effect is consistent with prior work on perceptual fluency, which reported higher confidence in decisions involving familiar rather than unfamiliar stimuli (Shaw, 1996). Finally, confidence ratings were found to be higher on Caucasian-Asian continua $(M=4.86, S D$ - 1.63) than on Caucasian-North African continua $(M=4.64, S D=1.66), F(1,45)=$ $5.82, p<.02$.

Table 4 Categorization of Mixed-Race Faces as a Function of Level of Morphing (Study 2)

\begin{tabular}{lccc} 
& \multicolumn{3}{c}{ Level of morphing (\%) } \\
\cline { 2 - 4 } Continua & 30 & 50 & 70 \\
\hline Caucasian-Asian: Categorization as Asian & & & \\
$M$ & 0.23 & 0.67 & 0.89 \\
$S D$ & 0.25 & 0.28 & 0.18 \\
Caucasian-North African: Categorization as & & & \\
North African & & & \\
$M$ & 0.46 & 0.56 & 0.68 \\
$S D$ & 0.34 & 0.25 & 0.24 \\
\hline
\end{tabular}

Note. Values refer to the proportion of faces assigned to the low end (i.e., Caucasian) versus high end (i.e., Asian and North African) of the continua.

\section{Discussion}

Study 2 extended beyond the first study by adding a new experimental setting and new continua types. Participants' recollection of moderately ambiguous faces was distorted toward more typical faces, and this effect proved to be stronger in recollecting own-race (i.e., 30\%) than other-race (i.e., 70\%) faces. As for the most ambiguous faces $(50 \%)$, no systematic distortion was observed, and the social information attached to the face did not influence recollection. This absence of effect can hardly be attributed to corrective processes, because participants were unable to recall face-label associations at the identification time. We elaborate on these points in the General Discussion section.

\section{Study 3}

Results from Studies 1 and 2 support the view that category-consistent accentuations emerge in recollecting ethnically ambiguous faces. However, a critical reader may say that our morphing procedure confounded ethnicity with individual identity. Admittedly, morphing two faces from different ethnicities not only creates faces ambiguous according to their ethnicity, but it also alters the distinctiveness of individual characteristics in these faces. Several studies have reported higher accuracy in the recollection of distinctive faces (e.g., Going \& Read, 1974; Valentine, 1991; Valentine \& Bruce, 1986a, 1986b), and one may assume that people encode distinctive features when learning a new face. As a consequence, it may be that participants in Studies 1 and 2 selected the face that displayed the most distinctive features when trying to remember which face they had seen. For instance, participants presented with a $70 \%$ face may have selected $80 \%$ more than $60 \%$ distractors because the prevalence of distinctive features was relatively higher in $80 \%$ faces. ${ }^{4}$ The latter process may or may not have anything to do with ethnicity.

Results obtained on association scores in Studies 1 and 2 suggest that categorization effects truly underlay the

\footnotetext{
${ }^{4}$ We thank two anonymous reviewers for having raised this possibility to us.
} 
memory distortions we obtained. However, the possibility exists that participants consciously matched their categorization reports with the identification choice(s) they had made. In our view, it is hardly likely that such a rationalization process operated in Study 2, in which eight identification choices were made but only one categorization decision was reported for each face. It is also hardly likely that participants in Study 1 justified their prior categorization of the face by consciously selecting a distractor that matched their original categorization report 1 week later. The rationalization account, although unlikely, cannot however be entirely dismissed. Indeed, distinctive faces, to some extent, may have attracted participants' choices. As an additional concern, the impact of categorization on association scores was significant for both the $30 \%$ (i.e., own-race) and the $70 \%$ (i.e., other-race) faces in Study 2, but these associations prove significant only when averaging across the $30 \%$ and $70 \%$ faces in Study 1 (which relied on a less powerful design). Therefore, we needed to examine (a) whether distortions toward more typical faces would operate over and above mere distortions toward distinctive faces and (b) whether they would do so for both the 30\% (own-race) and 70\% (other-race) faces.

These issues were examined in Studies 3 and 4, in which we predicted that memory distortions toward source faces would be larger on mixed-race (i.e., Caucasian-Asian) than same-race (Caucasian-Caucasian) continua. Obtaining evidence for the later prediction would provide strong support for the view that accentuation toward more typical faces operates over and above accentuation toward distinctive face features. As for the mixed-race continua, we considered only Caucasian-Asian faces in these studies. We did so in order to limit the number of experimental conditions and because the continua type did not play a key role in Study 2.

\section{Method Participants and Design}

Thirty-two Caucasian undergraduate students at the Catholic University of Louvain took part in the experimental session for partial course credit. The design was a 2 (morphing: $30 \%$ vs. $70 \%$ faces) $\times 2$ (continua type: Caucasian-Caucasian vs. Caucasian-Asian) $\times 2$ (distractor type: closer to the $0 \%$ vs. the $100 \%$ source face) experimental design, with continua and distractor type as within-participants factors. Participants were run individually and were randomly assigned to one level of the morphing factor.

\section{Materials}

Eight continua were used in this study. Four of them were the four Caucasian-Asian continua used in Study 2, and four of them were Caucasian-Caucasian continua created following the same procedure as for the CaucasianAsian continua. An example of a Caucasian-Caucasian continuum can be seen in Appendix C. The target faces were located at $30 \%$ or $70 \%$ on the continua. As for Studies 1 and 2, distractors were $10 \%$ and $20 \%$ closer to the $0 \%$ and $100 \%$ source faces. The pictures were presented in color, except for two continua for which a color version was not available. Because we focused on the undirected categorization process in Studies 3 and 4, social information or ethnic labels were never attached to the faces.

\section{Procedure}

On their arrival at the laboratory, participants were seated in front of a Macintosh 400Mhz PowerBook computer. All instructions were communicated via the computer program. The procedure was the same as for Study 2 , except that participants were presented with four instead of eight faces: two Caucasian-Asian faces and two Caucasian-Caucasian faces. ${ }^{5}$ As a result, participants evaluated 32 (instead of 64) pairs in the identification task. At the end of the experimental session, participants were thanked and fully debriefed.

\section{Results}

As in Study 2, participants' identifications on bom distractor types were receded as scores ranging from 0 (always correct) to 1.5 (always wrong). Consistent with the prediction that accentuation toward source faces would be larger on mixed-race than same-race continua, we submitted these scores to a contrast coding, with weights of $+2,-2,+1$, and -1 , respectively for selections of distractors toward the $0 \%$ Caucasian-Asian source face $(+2)$, the $100 \%$ Caucasian-Asian source face $(-2)$, the $0 \%$ Caucasian-Caucasian source face $(+1)$, and the $100 \%$ Caucasian-Caucasian source face $(-1)$.

We anticipated that this contrast would be moderated by the morphing factor. This global interaction reflects our

\footnotetext{
${ }^{5}$ In order to keep the faces involved in same-race and mixed-race continua as similar as possible, the $0 \%$ source faces were yoked across the continua types. So a Caucasian face was morphed either with another Caucasian face or with another Asian face. This constrained us to expose each participant to only tour faces. Two versions of the program were used, however, which involved the presentation of eight-face continua in total. The latter factor did not moderate the results and is not discussed further.
} 
prediction for larger (smaller) accentuations of the 30\% faces toward the $0 \%$ (100\%) source face and larger (smaller) accentuations of the $70 \%$ faces toward $100 \%(0 \%)$ source faces on mixed-race than same-race continua. This global interaction was not significant, $F(1,31)=2.47, p<.13$. This was essentially due to unexpected findings obtained in the recollection of the $30 \%$ faces. Indeed, although our predictions were fully supported in the case of $70 \%$ faces, they were unsubstantiated for the $30 \%$ faces. Below, we examine separately the distortion patterns that emerged in recollecting the $70 \%$ (i.e., other-race) and $30 \%$ (i.e., own-race) faces.

\section{Identifications of the $70 \%$ Faces}

The above contrast coding on the distortion score was applied to identifications of the $70 \%$ faces. This contrast was significant, $F(1,15)=4.58, p<.05$, revealing a greater ratio of memory distortions toward the $100 \%$ than toward the $0 \%$ source faces on the Caucasian-Asian than on the Caucasian-Caucasian continua. The full pattern of means is reported in Table 5, and this pattern nicely reflects our prediction that accentuations toward typical faces emerge over and above accentuations toward distinctive faces.

As in Study 2, we analyzed associations between participants' categorizations and recollections of the $70 \%$ Caucasian-Asian faces (an assimilative association was given a +1 value, a contrastive association received a -1 value, and we averaged across the various mixed-race faces). As expected, distortions in recollecting these faces were more assimilative than contrastive $(M=0.50, S D=0.65), F(1,13)=8.27, p<.02$.

Participants also appeared to be marginally more confident when identifying a Caucasian-Asian face $(M=4.89$, $S D=1.35)$ than a Caucasian-Caucasian face $(M=4.42, S D=1.26), F(1,15)=3.78, p=.07$. As in Study 2 , they were also more confident when making correct $(M=4.92, S D=1.22)$ than incorrect $(M=4.04, S D=1.42)$ identifications, $F(1,15)=15.46, p<.01$.

\section{Identifications of the $30 \%$ Faces}

For the $30 \%$ faces, the expected linear contrast was not significant, $F(1,15)<1$. This unexpected result is discussed below, and it receives further attention in Study 4.

Table 5 Mean Recollection Distortion of 70\% Faces as a Function of Distractor Type and Continua Type (Study 3)

\begin{tabular}{lcc}
\hline & \multicolumn{2}{c}{ Continua type } \\
\cline { 2 - 3 } Distractor type & Caucasian-Caucasian & Caucasian-Asia \\
\hline Caucasian & & \\
$M$ & 0.37 & 0.23 \\
$S D$ & 0.30 & 0.18 \\
Caucasian-Asian & & \\
$M$ & 0.49 & 0.59 \\
$S D$ & 0.46 & 0.38 \\
\hline
\end{tabular}

Note. Values range from 0 (correct identifications on all identification trials) to 1.5 (incorrect identifications on all identification trials).

\section{Discussion}

Study 3 shows that memory distortions toward ethnic prototypes emerge over and above mere accentuations toward extreme faces in the case of other-race (i.e., 70\% Asian) faces. This is an important finding, which is fully consistent with our line of reasoning. We failed, however, to obtain evidence for category-consistent accentuations in the case of own-race (i.e., 30\% Asian) faces. This absence of a result may have resulted incidentally from the use of a within-participants design in Study 3. Indeed, we surmise that the withinparticipants manipulation of the continua type factor decreased the perceived (Caucasian) typicality of the $30 \%$ Caucasian-Asian faces relative to the $30 \%$ Caucasian-Caucasian faces.

In order to further examine the latter possibility, we ran a fourth study in which we examined memory distortions for 30\% Caucasian-Caucasian and Caucasian-Asian faces while manipulating the continua type factor between participants. In this case, we predicted that the accentuation toward ethnic prototypes would emerge over and above mere accentuation toward extreme faces. In other words, we predicted larger memory distortions toward 
the $0 \%$ face on Caucasian-Asian than on Caucasian-Caucasian continua.

\section{Study 4}

\section{Method Participants and Design}

Fifty-five Caucasian undergraduate students at the Catholic University of Louvain participated in the experimental session for partial course credit. The design was a 2 (continua type: Caucasian-Caucasian vs. Caucasian-Asian) $\times 2$ (distractor type: closer to the $0 \%$ vs. the $100 \%$ source face) experimental design, with distractor type as a within-participants factor. Participants were studied in group sessions of 10 persons and were randomly assigned to one level of the continua type factor.

\section{Materials}

The same (30\%) faces used in Study 3 were used in this study.

\section{Procedure}

On their arrival at die laboratory, participants were seated in front of a PowerPC computer with a Trinitron monitor. All instructions were communicated via the computer program. The procedure was the same as in Study 3. Because the continua type factor was manipulated between participants, four same-race or mixed-race faces could be presented to each participant instead of just two as was the case in Study 3. Participants were fully debriefed and thanked at the end of the experimental session.

\section{Results}

As in Studies 2 and 3, answers on the identification trials were recoded into scores ranging from 0 (always correct) to 1.5 (always wrong) for answers on both distractor types. These scores were submitted to a 2 (distractor type: closer to the $0 \%$ vs. the $100 \%$ source face) $\times 2$ (continua type: Caucasian-Asian vs. CaucasianCaucasian) mixed ANOVA. As predicted, the interaction between these factors was significant, $F(1,53)=5.99$, $p<.02$, with greater memory distortions toward the $0 \%$ source faces than toward the $100 \%$ source faces on the Caucasian-Asian than on the Caucasian-Caucasian continua. The full pattern of means is reported in Table 6 . Again, this pattern fully supports our prediction that accentuations toward more typical faces emerge over and above accentuations toward more distinctive faces.

As in Studies 2 and 3, we analyzed associations between participants' categorizations and recollections of the Caucasian-Asian faces (an assimilative association was given a +1 value, a contrastive association received a -1 value, and we averaged across identifications of the various faces). Again, assimilative associations were significantly greater than contrastive ones $(M=0.60, S D=0.63), F(1,20)=19.3, p<.01$.

The level of participants' confidence was about the same as that observed in Studies 2 and $3(M=5.74, S D=$ 1.28). It was significantly higher for correct identifications $(M=5.92, S D=1.29)$ than for incorrect identifications $(M=5.28, S D=1.35), F(1,54)=50.55, p<.01$. Again, we attribute this effect to the greater familiarity of the target face that emerged throughout the identification task.

Table 6 Mean Recollection Distortion of 30\% Faces as a Function of Distractor Type and Continua Type (Study 4)

\begin{tabular}{|c|c|c|}
\hline \multirow[b]{2}{*}{ Distractor type } & \multicolumn{2}{|c|}{ Continua type } \\
\hline & Caucasian-Caucasian & Caucasian- \\
\hline Caucasian & & \\
\hline$M$ & 0.43 & 0.52 \\
\hline$S D$ & 0.21 & 0.26 \\
\hline Caucasian-Asiar & & \\
\hline$M$ & 0.32 & 0.21 \\
\hline$S D$ & 0.17 & 0.16 \\
\hline
\end{tabular}

Note. Values range from 0 (correct identifications on all identification trials) to 1.5 (incorrect identifications on all identification trials). 


\section{General Discussion}

In the four studies reported here, we obtained evidence for accentuation effects in face memory. Participants who were presented with faces moderately typical of Caucasian people tended to recollect these faces as more Caucasian than they actually were. Similarly, participants who were presented with faces moderately typical of North African or Asian people tended to recollect these faces as more North African or Asian than they actually were. Importantly, in all studies, memory distortions were more likely to be consistent than inconsistent with the categorization of the face, and the categorization of the face predicted memory distortions 1 week later in Study 1.

Also important, these findings were obtained in various experimental settings that varied the nature and number of faces to be learned as well as the nature of the encoding, distracting, and identification tasks. They were also obtained with substantial levels of confidence. Finally, Studies 3 and 4 provided evidence that memory distortions toward more typical faces emerged over and above mere distortions toward distinctive faces. Incidentally, the comparison of results obtained for the $30 \%$ faces in Studies 3 and 4 suggests that recollective biases may partly depend on the face anchors that are activated in the setting. The later finding would deserve further examination in the context of studies that directly manipulate face anchors and assess the role that this factor may play in recollecting both own-race and other-race faces. We now elaborate on the theoretical and practical implications of these findings.

\section{Undirected and Directed Categorization Effects: The Impact of Information Inherent and External to Faces}

The present findings are consistent with previous work conducted on reconstructive memory, in particular with Huttenlocher et al.'s $(1988,2000)$ model that was briefly outlined in the introduction of this article. This model posits that people's reports from episodic memory combine fine-grained and categorical information hierarchically encoded about stimuli and that this process results in the adjustment of people's recollections toward more typical values of the category. The findings obtained in the four studies reported here nicely fit this notion.

Although strong evidence was obtained for the impact of the features inherent in the faces, social information attached to these faces did not influence participants' recollection. Below, we discuss the conditions under which such influence may emerge.

\section{Increased Applicability of the Social Information}

Social information attached to ethnically ambiguous faces may be found to play a role in the recollection process when the consistency between the physical features of a face and the social information attached to it is maximized. Even then, the range of influence the social information garners may be narrower than expected. In the present studies, the $30 \%$ and $70 \%$ faces were immune to social information influences (we think because this information was highly redundant with the spontaneous categorization of the faces), and the $50 \%$ faces apparently faced an applicability issue. At first sight, the lack of applicability of the social information to the $50 \%$ faces may seem inconsistent with evidence showing that races are perceived categorically (Levin \& Beale, 2000). However, participants in categorical perception studies randomly assign, or learn to assign, extremely ambiguous faces into imposed ethnic categories. This effect, we think, does not directly speak to the influence that social information may have in the unconstrained classification and recollection of extremely am biguous faces.

\section{Implicit Learning}

The possibility exists that some participants in Study 1 corrected for mental contamination of their identification by the social information blatantly attached to the faces, whereas the distraction task used in Study 2 was so demanding that it interfered with the memory trace for the face-label associations, both at the explicit and implicit level. The learning task used in Study 2 was such that it required participants to encode face information along with the ethnic label attached to it. It may be, however, that such a procedure was too weak to allow an implicit learning of these associations. Ideally, the impact of the social information may be appreciated in experimental settings that test for both the explicit and implicit memory of the face-label associations at the identification stage. The impact of the social information might emerge when the retrieval of face-label associations is precluded at the identification time, whereas the implicit learning of these associations is guaranteed. The use of implicit learning procedures involving the repeated pairing of face stimuli with social information may, however, have little relevance for eyewitness identifications, because most eyewitness 
circumstances involve unique exposures to a crime scene.

\section{Explanation at the Encoding}

Social information may also distort people's memory of faces toward more typical faces when the situation requires that people attend to and actively process facial features in line with the social information communicated about the face. This possibility is consistent with the work by Halberstadt and Niedenthal (2001) discussed in the introduction of this article. Procedures requiring that participants actively generate categoryconsistent explanations about a face at the encoding may, however, increase the risk for experimental demands.

\section{Naive Theories}

Recently, Eberhardt et al. (2003) demonstrated that the recollection of extremely ambiguous Caucasian-African American faces is sensitive to an interaction between participants' naive theories and the social information attached to these faces. Specifically, "entiteist" individuals assimilate their recollection of the faces to the social information attached to them, whereas incrementalist individuals contrast their recollection away from this information (for a general discussion on the role of entity and incremental theories on person perception, see Levy, Plaks, \& Dweck, 1999). The result is the absence of systematic distortion in the recollection of extremely ambiguous faces when perceivers' naive theories are not taken into account. Because these naive theories were not addressed in the present research, we can only speculate on the role that this factor may have played here. Further research would, however, benefit from paying closer attention to this issue.

\section{Distortions in Recollections and Distortions in Decisions}

An issue worth addressing is whether our effects were due to distortions in participants' face memory or to strategic guesses in the identification task. According to the first view, participants' memory traces were distorted toward more typical exemplars as they encoded or recollected the faces along with the categorical label spontaneously associated with these faces. According to the second view, participants did not distort their memory traces of the faces in any systematic way but strategically relied on the categorical label spontaneously associated with the faces in order to maximize their ability to guess correctly in the identification task. In sum, these views would, respectively, posit that the distortions obtained in the present studies represented truthful reports of distorted memories or untruthful reports of undistorted memories.

The confidence ratings we obtained suggest that the first process (memory distortion) was more likely to operate than the second one (strategic guess). Assuming that participants merely guessed about their decisions, it is very unlikely that they were so confident in their identifications. Both processes may, however, have operated to some extent in producing identification distortions. Their respective influence may be assessed by conducting a signal detection analysis on old-new judgments on discrete face exemplars.

Finally, assuming that categorization distorted participants' memory traces, one may wonder whether this effect emerged as participants encoded the fine-grained information about the face or, alternatively, as they recollected this information from memory. Definite answers to this question are clearly beyond the scope of this particular research endeavor.

\section{Accentuation Toward More Typical Own-Race and Other-Race Faces}

In the introduction to the present article, we noted that our experimental procedure allowed us to examine whether accentuations toward more typical faces would be larger in identifying own-race or other-race faces. Given the current state of the literature on face representations, conflicting predictions could be made on this issue.

The literature on the other-race effect has demonstrated that people are generally better at recognizing own-race than other-race faces. According to Valentine's (1991) model of face representation, faces are encoded along discriminant dimensions. These dimensions are abstracted from concrete face exemplars encountered in everyday life, which are more likely to be own-race than other-race. Unbalanced exposure to own-race and other-race faces leads to relatively more discriminant dimensions in encoding own-race than other-race faces, in turn leading to more precise encodings of the fine-grained information for own-race faces (see also Furl, Phillips, \& O'Toole, 2002). Given the existence of more precise encoding for own-race faces, one may have expected accentuation effects to be smaller for own-race $(30 \%)$ than for other-race $(70 \%)$ faces in the present studies. In terms of Hutten-locher et al.'s (1988, 2000) model, the better encoding of the fine-grained information for own- 
race faces might have resulted in a lower impact of the categorical information in the case of own-race identifications.

Contrary to the above view, the findings we obtained in Study 2 suggest that, if anything, accentuation toward more typical faces was smaller for other-race faces than for own-race faces. How can this finding be accounted for? In our view, this pattern of results is consistent with the idea that prototypes serve as cognitive reference points that function as magnets (Rosch, 1975). Magnet effects correspond to a lower discriminability and a presumed shrinkage of psychological space around reference points (Quinn, 2000). These effects have been evidenced in a number of studies that revealed asymmetries in similarity judgments: People tend to consider nonprototypic stimuli as more similar or closer to prototypic stimuli than the opposite (Kuhl, 1991; Rosch, 1975; Tversky, 1977).

Recently, Levin (2000) used the magnet principle as a proposition to account for the existence of better discrimination scores in discriminating between morphed faces located on the African American side than on the Caucasian side of an African American-Caucasian continuum of faces. Because people hold stronger representations for own-race than for other-race prototypes (because of the high encounter of own-race faces), a stronger magnet effect toward own-race than toward other-race prototypes may emerge. This may explain why enhanced distortions toward own-race than other-race prototypes tended to emerge in the present studies. Levin also suggested that the discrimination advantage for other-race faces on mixed-race continua may be due to an asymmetry in features processed for own-race and other-race faces. According to this feature-selection hypothesis, when encoding other-race faces, people emphasize information that specifies racial features at the expense of individuating features. This process may also apply to the results we obtained here. Specifically, distortions should be less likely to emerge on the other-race side of mixed-race continua if, as suggested by Levin (2000), racial features of other-race faces are more closely attended to in the context of these continua.

\section{Practical Implications for Eyewitness Testimony}

The quality of face memory is crucial in trials involving eyewitness identifications. Indeed, mistaken identification is responsible for more wrongful convictions of innocent people than all other causes combined (Wells, Small, Penrod, Malpass, Fulero, \& Brimascombe, 1998). Recently, the introduction of DNA analysis has permitted the exoneration of numerous innocent people, most of them having been identified by eyewitnesses. This problem is so important that the researchers established guidelines for procedures of eyewitness identifications. In 1999, the American National Institute of Justice published a guide (Technical Working Group for Eyewitness Evidence, 1999) explaining the procedures that can be used to obtain the most reliable and accurate information from eyewitnesses. As explained in the introduction of this guide (Technical Working Group for Eyewitness Evidence, 1999, p. III), even the most honest and objective people can make mistakes in recalling and interpreting what they witnessed.

At the beginning of the article, we emphasized that recollection distortions toward prototypes may be of interest for scholars working on eyewitness testimony. Indeed, we suspect that such distortions may induce a supplementary bias threatening the accuracy of face memory. The experimental setting used in the present set of studies has little correspondence with real lineup situations, because one would hardly imagine having morphea individuals involved in a lineup. On the basis of the findings reported here, however, it is worth examining whether accentuation effects may lead an eyewitness to falsely identify a person whose facial features look the most typical of the ethnic category spontaneously associated with the culprit's face.

In order to address that important issue, it seems important to distinguish between situations in which the culprit is or is not present in the lineup. When the culprit is present in the lineup, we believe that the risk for misidentifications due to an accentuation effect will be relatively low. Indeed, if an eyewitness had the opportunity to encode the face of a culprit fairly well, it seems unlikely that accentuation effects will lead him or her to select a distractor instead of the actual culprit who is present in the identification task. One should note, however, that the risk for such confusions may increase when the encoding of the culprit's face is poor or as time length increases between the crime and the identification procedure, both factors leading to a loss of fine-grained information about the face.

In many situations, eyewitnesses also have to make a decision in a lineup that does not involve the actual culprit. Under these circumstances, the risk for misidentifications due to accentuation effects may be higher. More specifically, the risk is that eyewitnesses identify the person in the lineup who most resembles the culprit relative to other members of the lineup (Wells, 1984), especially if that person is more typical of the culprit's ethnic category than are the other distractors. Here again, misidentifications toward category prototypes may be 
enhanced under conditions of poor memory for the culprit's face. This is not only because categorical information will have a higher impact in such conditions, but also because the relative resemblance bias will be reduced. Clearly, if one has a very poor memory of a culprit's face, then the risk for relative judgments should decrease and the use of category-based judgments should increase.

More generally, one should note mat empirical work examining the above issues should be able to manipulate independently the similarity and typicality factors. This goal may be difficult to achieve: By definition, the more similar two faces are, the less different they should be with regard to typicality. Nevertheless, we think that intense pretest work may provide experimental materials that vary the similarity and typicality factors independently and ecologically. Studies conducted with such materials may reveal that distortions toward prototypes evidenced in the present experiments generalize to situations where faces from many different individuals are involved in a lineup.

\section{Conclusions}

In their recent overview of memory distortion effects, Roediger and McDermott (2000) noted that "people would like to believe that their memories are more or less accurate renditions of the experiences that occurred to them in the past" (p. 149). Experiencing a clash between the reality and one's recollection of it can be a most troubling experience indeed, especially when false memories are held with a high level of confidence.

Under certain circumstances, people will eventually notice their confusion. When that is the case, they will have the opportunity to correct for their decisions and judgments, and their confusion will only result in pleasant anecdotes to be reflected on or socially shared on later occasions. In other circumstances, however, people will be intimately convinced about the accuracy of their false memories, and this could lead to tragic consequences, either for themselves or for their social environment.

The present set of studies provides original evidence for the emergence of such memory distortions in the recollection of highly complex, multifaceted, and socially relevant stimuli: human faces from various ethnicities, and we have discussed the social implications of our findings in the context of eyewitness testimony. Along with recent work conducted on related issues (e.g., Lenton, Blair, \& Hastie, 2001; Macrae, Schloerscheidt, Bodenhausen, \& Milne, 2002), we hope that the present work will thrust the issue of memory illusions onto a more central stage in contemporary social psychology.

\section{Acknowledgement}

We are grateful to Raymond Bruyer, Jamin Halberstadt, Jacques-Philippe Leyens, and Vincent Yzerbyt for their valuable comments on an earlier version of this article.

\section{References}

Bartlett, F. C. (1932). Remembering: A study in experimental and social psychology. Cambridge, England: Cambridge University Press.

Begg, I. M., Anas, A., \& Farinacci, S. (1992). Dissociation of process in belief: Source recollection, statement familiarity, and the illusion of truth. Journal of Experimental Psychology: General, 121, 446-458.

Blair, I. V., Judd, C. M., Sadler, M. S., \& Jenkins, C. (2002). The role of Afrocentric features in person perception: Judging by features and categories. Journal of Personality and Social Psychology. 83, 5-25.

Brewer, W. F., \& Nakamura, G. V. (1984). The nature and functions of schémas. In R. S. Wyer \& T. K. Srull (Eds.), Handbook of social cognition (Vol. 1, pp. 119-160). Hillsdale, NJ: Erlbaum.

Corneille, O., Klein, O., Lambert, S., \& Judd, C. M. (2002). On the role of familiarity with units of measurement in producing categorical accentuation: Tajfel and Wilkes (1963) revisited and replicated. Psychological Science, 4, 380-383.

Dodson, C. S., Johnson, M. K., \& Schooler, J. W. (1997). The verbal overshadowing effect Why descriptions impair face recognition. Memory and Cognition, 25, 129-139.

Eberhardt J. L., Dasgupta, N., \& Banaszynski, T. L. (2003). Believing is seeing: The effect of racial labels and implicit beliefs on face 
Published in: Journal of Personality and Social Psychology (2004), vol. 86, iss. 2, pp. 236-250.

Status: Postprint (Author's version)

perception. Personality and Social Psychology Bulletin. 29, 360-370.

Fiedler, K. (1993). Constructive processes in person cognition. British Journal of Social Psychology, 32, 349-364.

Fiedler, K., Armbruster, T., Nickel, S., Walther, E., \& Asbeck, J. (1996). Constructive biases in social judgment: Experiments on the selfverification of question contents. Journal of Personality and Social Psychology, 71, 861-873.

Furl, N., Phillips, J. P., \& O'Toole, A. J. (2002). Face recognition algorithms and the other-race effect: Computational mechanisms for a developmental contact hypothesis. Cognitive Science, 26, 797-815.

Going, M., \& Read, J. D. (1974). The effect of uniqueness, sex of subject, and sex of photograph on facial recognition. Perceptual and Motor Skilh, 39, 109-110.

Gryphon Software Corporation. (1992-1994). Morph TM 2.5 [Computer software], San Diego, CA: Author.

Halberstadt, J. B., \& Niedentlial, P. M. (2001). Effects of emotion concepts on perceptual memory for emotional expression. Journal of Personality and Social Psychology, 81, 587-598.

Higgins, E. T. (1996). Knowledge activation: Accessibility, applicability, and salience. In E. T. Higgins \& A. W. Kruglanski (Eds.), Social psychology: Handbook of basic principles (pp. 133-168). New York: Guilford Press.

Huttenlocher, J., Hedges, L. V., \& Duncan, S. (1988). Categories and particulars: Prototype effects in the estimating spatial location Psychological Review, 98, 352-376.

Huttenlocher, J-, Hedges, L. V., \& Vevea, J. L. (2000). Why do categories affect stimulus judgment? Journal of Experimental Psychology: General. 129, 220-241.

Klauer, K..-C., \& Wegener, I. (1998). Unraveling social categorization in the "Who said what?" paradigm. Journal of Personality and Social Psychology. 75, 1155-1178

Kuhl, P. K. (1991). Human adults and human infants show a perceptual magnet effect for the prototypes of speech categories, monkeys do not. Perception and Psychophysics, 50, 93-107.

Lenton, A. P., Blair, I. V., \& Hastie, R. (2001). illusions of gender: Stereotypes evoke false memories. Journal of Experimental Social Psychology, 37, 3-14.

Levin, D. T. (2000). Race as a visual feature: Using visual search and perceptual discrimination tasks to understand face categories and the cross-race recognition deficit. Journal of Experimental Psychology: General. 129, 559-574.

Levin, D. T., \& Beale, J. M. (2000). Categorical perception occurs in newly learned faces, other-race faces, and inverted faces. Perception and Psychophysics, 62, 386-401.

Levy, S. R., Plaks, J. E., \& Dweck, C. S. (1999). Modes of social thought: Implicit theories and social understanding. In S. Chaiken \& T. Trope (Eds.), Dual-process theories in social psychology (pp. 179-202). New York: Guilford Press.

Livingston. R. W., \& Brewer, M. B. (2002). What are we really priming? Cue-based versus category-based processing of facial stimuli. Journal of Personality and Social Psychology, 82, 5-18.

Loftus. E. F. (1975). Leading questions and the eyewitness report. Cognitive Psychology, 7, 560-572.

Loftus, E. F., Miller, D. G., \& Bums, H. J. (1978). Semantic integration of verbal information into a visual memory. Journal of Experimental Psychology: Human Learning and Memory. 4, 19-31.

Loftus, E. F., \& Palmer, J. P. (1974). Reconstruction of automobile destruction: An example of the interaction between language and memory. Journal of Verbal Learning and Verbal Behavior, 13, 585-589.

MacLin, O. H., \& Malpass, R. S. (2001). Racial categorization of faces: The ambiguous race face effect. Psychology, Public Policy, and Law, 7, 98-118.

Macrae, C. N., Schloerscheidt, A. M., Bodenhausen, G. V., \& Milne. A. B. (2002). Creating memory illusions: Expectancy-based processing and the generation of false memories. Memory, 10, 63-80.

Maddox, K. B., \& Gray, S. A. (2002). Cognitive representations of Black Americans: Reexploring the role of skin tone. Personality and Social Psychology Bulletin, 28, 250-259.

Malpass, R. S., \& Kravitz, J. (1969). Recognition for faces of own and other races. Journal of Personality and Social Psychology, 13, 333 334. 
Published in: Journal of Personality and Social Psychology (2004), vol. 86, iss. 2, pp. 236-250.

Status: Postprint (Author's version)

Posner, M. I., \& Keele, S. W. (1968). On the genesis of abstract ideas. Journal of Experimental Psychology, 77, $353-363$.

Quinn, P. C. (2000). Perceptual reference points for form and orientation in young infants: Anchors or magnets7 Perception and Psychophysics, 62, 1625-1633.

Reber, R., \& Schwarz, N. (1999). Effects of perceptual fluency on judgments of truth. Consciousness and Cognition, 8, 338-342.

Roediger, H. L., \& McDermott, K. B. (2000). Distortions of memory. In E. Tulving \& F. I. M. Craik (Eds.), The Oxford handbook of memory (pp. 149-162). New York: Oxford University Press.

Rosen, E. H. (1975). Cognitive reference points. Cognitive Psychology, 1, 532-547.

Shaw, J. S., III. (1996). Increases in eyewitness confidence resulting from postevent questioning. Journal of Experimental Psychology: Applied, 2, 126-146.

Tajfel, H., \& Wilkes, A. L. (1963). Classification and quantitative judgment. British Journal of Psychology, 54, $101-114$.

Taylor, S. E., Fiske, S. T., Etcoff, N. L., \& Ruderman, A. (1978). Solo status as a psychological variable: The power of being distinctive. Journal of Personality and Social Psychology, 36, 778-793.

Technical Working Group for Eyewitness Evidence (1999). Eyewitness evidence: A guide for law enforcement. Washington, DC: U.S, Department of Justice, Office of Justice Programs.

Trope, Y. (1986). Identification and inferential process in dispositional attribution. Psychological Review, 93, $239-257$.

Trope, Y.. \& Cohen, O. (1989). Perceptual and inferential determinants of behavior-correspondent attributions. Journal of Experimental Social Psychology. 25, 142-158.

Trope, Y., Cohen, O., \& Maoz, Y. (1988). The perceptual and inferential effects of situational inducements on dispositional attribution. Journal of Personality and Social Psychology, 55, 165-177.

Tversky, A. (1977). Features of similarity. Psychological Review, 84, 327-352.

Valentine, T. (1991). A unified account of the effects of distinctiveness, inversion, and race in face recognition. Quarterly Journal of Experimental Psychology: Human Experimental Psychology, 43, 4161-4204.

Valentine, T., \& Bruce, V. (1986a). The effect of distinctiveness in recognizing and classifying faces. Perception, 15, 525-535.

Valentine, T., \& Bruce, V. (1986b). Recognizing familiar faces: The role of distinctiveness and familiarity. Canadian Journal of Psychology, 40, 300-305.

Wells, G. L. (1984). The psychology of lineup identifications. Journal of Applied Social Psychology, 14, 89-103.

Wells, G. L., Small. M., Penrod, S., Malpass, R. S., Fulera, S. M., \& Brimascombe, C. A. E. (1998). Eyewitness identification procedures: Recommendations for lineups and photospreads. Law and Human Behavior, 22, 1-39.

Wilson, T. D., \& Brekke. N. (1994). Mental contamination and mental correction: Unwanted influences on judgments and evaluations. Psychological Bulletin, 116. 117-142. 


\section{RECOLLECTION OF FACES}

Appendix A

Example of Caucasian-North African Continuum

Figure A1. NA = North African
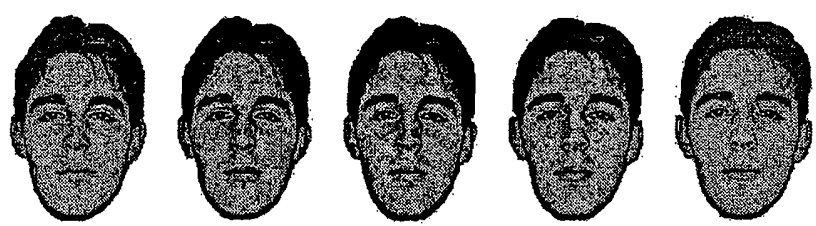

$0 \% \mathrm{NA}$

$10 \% \mathrm{NA}$

$20 \%$ NA

30\% NA

40\%.NA
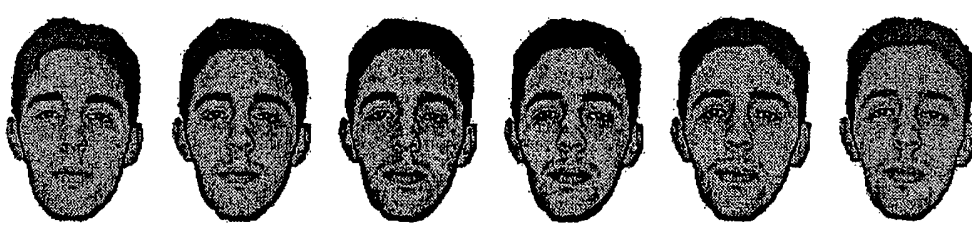

$50 \%$ NA

$60 \%$ NA

$70 \%$ NA

$80 \%$ NA

90\% NA

$100 \%$ NA

Appendix B

Example of Caucasian-Asian Continuum

Figure B1. AS = Asian.
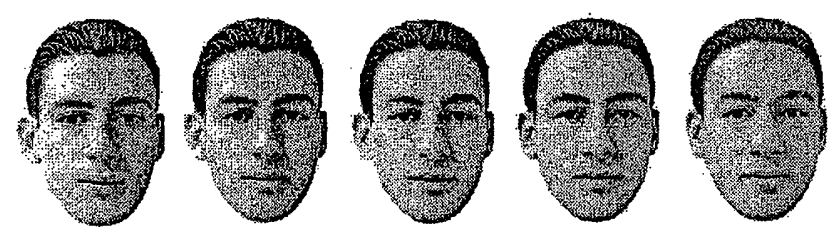

0\% AS

$10 \%$ AS $20 \%$ AS

$30 \%$ AS

$40 \%$ AS

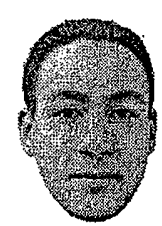

$50 \%$ AS

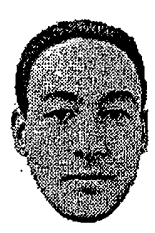

$60 \%$ AS

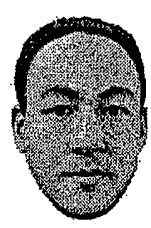

$70 \%$ AS

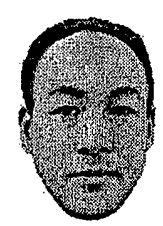

$80 \%$ AS

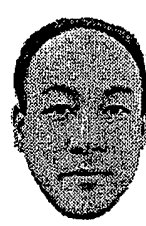

$90 \%$ AS

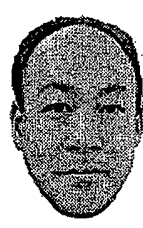

$100 \%$ AS

\section{Appendix C}

Example of Caucasian-Caucasian Continuum

Figure C1. 
Published in: Journal of Personality and Social Psychology (2004), vol. 86, iss. 2, pp. 236-250.

Status: Postprint (Author's version)
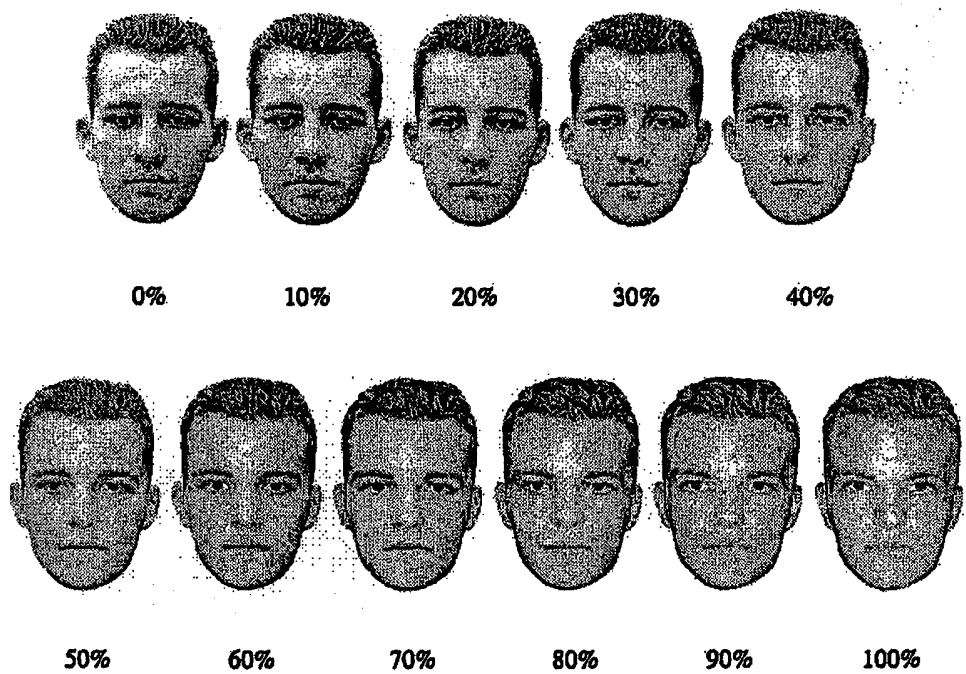